\title{
MANEJO DE LOS DESPRENDIMIENTOS DE RETINA REGMATÓGENOS URGENTES EN EL SISTEMA NACIONAL DE SALUD DE ESPAÑA. PROYECTO RETINA 2
}

\author{
URGENT RETINAL DETACHMENT MANAGEMENT BY THE \\ NATIONAL HEALTH SYSTEM OF SPAIN. PROJECT RETINA 2
}

ROJAS J ${ }^{1}$, FERNÁNDEZ I², PASTOR JC ${ }^{3}$, GÓMEZ-ULLA F ${ }^{4}$, PIÑERO A ${ }^{5}$

\section{RESUMEN}

Objetivo: Establecer los problemas en el manejo de los desprendimientos de retina (DR) durante los fines de semana en el Sistema Nacional de Salud (SNS) español.

Métodos: Se envió una encuesta confidencial al jefe de Servicio de 217 hospitales del SNS. Los datos fueron almacenados en una base de datos Microsoft Access y analizados estadísticamente con Excel y Statgraphics. Las variables cualitativas fueron analizadas con el test de Chi-cuadrado y el test exacto de Fisher, y las cuantitativas con el test no paramétrico de Kruskall-Wallis.

Resultados: Se obtuvo una tasa de respuesta del $54,8 \%$ con mayor participación de hospitales docentes (HD). Los Comarcales (HC) se incluyeron en el grupo de los No Docentes $(\mathrm{HnD})$ por su patrón de respuestas similares. El 18\% de centros no tiene oftalmólogo de guardia. De los que sí disponen, el $36 \%$ tiene especialistas de vítreo retina de guardia.
ABSTRACT

Objective: To identify problems in the urgent management of retinal detachments (RD) in hospitals of the Spanish National Health System (NHS). Methods: A questionnaire was submitted confidentially to the heads of Ophthalmic Departments of 217 hospitals of the NHS to obtain information on the management of urgent RD during the last year. Data was stored in a Microsoft Access database and statistically analyzed by Excel and Statgraphics. Qualitative variables were analyzed by Chi-Square and Fisher exact tests and quantitative variables by the Kruskall-Wallis test.

Results: A global response rate of $54.8 \%$ was achieved with higher participation of the Teaching Hospitals (TH). District Hospital and non-Teaching Hospital responses were similar and grouped as non-TH. Eighteen percent of centers, mostly non$\mathrm{TH}$, had no ophthalmologist on duty. Thirty-six percent had a vitreoretinal specialist on call. Eighty

\footnotetext{
Recibido: 31/3/06. Aceptado: 26/3/07.

1 Licenciada en Medicina. Instituto de Oftalmobiología Aplicada. Grupo de Retina. Universidad de Valladolid. Becaria de la Fundación Carolina. Ministerio de Asuntos Exteriores y Cooperación.

${ }^{2}$ Licenciada en Estadística. Instituto de Oftalmobiología Aplicada. Grupo de Retina. Universidad de Valladolid. Becaria FIS.

3 Doctor en Medicina. Instituto de Oftalmobiología Aplicada. Grupo de Retina. Universidad de Valladolid.

4 Doctor en Medicina. Universidad de Santiago de Compostela.

5 Doctor en Medicina. Universidad de Sevilla.

Comunicación presentada en el LXXXI Congreso de la S.E.O. (Zaragoza 2005) y en el Congreso de la Sociedad Española de Retina y Vítreo (Barcelona 2006).

Realizado en parte con una subvención del Instituto de Salud Carlos III al proyecto PI 041701. FIS-FEDER. Con el apoyo de la Sociedad Española de Oftalmología.
}

Correspondencia:

Jimena Rojas

Ramón y Cajal, 7

Edificio Ciencias de la Salud. IOBA

47005 Valladolid

España

E-mail: rojasjimena@yahoo.com 
Más del $80 \%$ de los centros admite tener problemas para atender los DR urgentes. El 24\% no tiene acceso a quirófano de oftalmología; el $50 \%$ refiere problemas con Anestesia y el 22\% dispone de enfermeras de Oftalmología.

El $64 \%$ realiza vitrectomías y de ellos, el $77 \%$ atiende urgencias. El $90 \%$ de los centros reconoce que un paciente con DR superior y mácula no afectada tardaría más de 24 horas en intervenirse, aunque el $84 \%$ lo considera una práctica inadecuada.

Conclusión: A pesar de los sesgos y cuestiones metodológicas, se han identificado problemas del SNS relacionadas al manejo de los DR urgentes. Esto proporciona importante información para organizar una cobertura más eficiente.

Palabras clave: Desprendimiento de retina, urgencias, fin de semana, Sistema Nacional de Salud, España. percent of centres admitted to have problems handling urgent RD during weekends. Twenty-four had no ophthalmic surgical theatre available. Fifty percent refered to have problems having an anaesthesiologist available and only $22 \%$ had ophthalmic trained personnel (nurses) available.

Sixty-four percent of centres performed pars plana vitrectomy (PPV) and $77 \%$ of those were able to perform an urgent PPV. Ninety percent admitted that a patient with a macula-threatening RD occurring at the beginning of the week-end would not be treated until at least 24 hours had elapsed, although $84 \%$ considered this to be inadequate.

Conclusion: Despite the methodological problems and bias of this questionnaire, we did identify several important problems in the management of urgent RD by NHS hospitals. The data obtained provides useful information to enable the quality of the NHS care of RD to be improved, particularly that available at the week-end (Arch Soc Esp Oftalmol 2007; 82: 279-284).

Key words: Retinal detachment, emergencies, weekend-RD, National Health System, Spain.

\section{INTRODUCCIÓN}

Los desprendimientos de retina (DR) son una patología ocular considerada como grave, no sólo por los oftalmólogos sino por gran parte de la población, que además suele percibirla como un proceso que requiere un tratamiento urgente. A lo largo del pasado siglo esta entidad pasó de ser considerada como intratable (1), a mostrar, tras la cirugía, reaplicaciones anatómicas en más del $90 \%$ de los casos, según algunos autores (2). El desarrollo de técnicas que permiten mejores resultados anatómicos, ha aumentado la preocupación por la agudeza visual final. Uno de los factores que influyen en ese resultado funcional es el tiempo transcurrido entre la aparición de los síntomas y su tratamiento (3). De forma ideal, aquellos DR que amenazan la mácula deberían ser reparados dentro de las primeras 24 horas, y un DR con compromiso macular de 2 a 3 días de evolución debería ser operado dentro de las siguientes 48 horas (4) a su ingreso en un centro.

Otro factor importante es la reaplicación de la retina tras el primer intento, ya que generalmente se asocia a un pronóstico visual más favorable (5).
Además hay que tener en cuenta el impacto a nivel del coste sanitario que supone un DR que no ha podido ser resuelto en un primer procedimiento, sobre todo si desarrolla una vitreoretinopatía proliferante (VRP) (6).

Por estas y otras razones, en algunos países de nuestro entorno, en los que también existe un SNS, se han promulgado cambios en el manejo de estos casos estableciéndose unidades de referencia, que reciben un importante volumen de pacientes y con una sólida organización académica. Esto favorece el adecuado entrenamiento de los cirujanos de retina y una eficaz cobertura sanitaria de la población (7).

La realización de una encuesta de ámbito nacional, en el contexto de un proyecto que denominamos Retina 2, para obtener información sobre el manejo de la patología vitreoretiniana en España, nos ha dado la oportunidad de conocer aspectos interesantes sobre el manejo de los desprendimientos de retina urgentes.

En un trabajo previo, se expusieron las limitaciones metodológicas de esta encuesta y los resultados generales (8). La finalidad del presente trabajo es la de exponer los resultados relacionados con el manejo de la patología vitreorretiniana urgente y los DR que ocurren durante los fines de semana. 


\section{SUJETOS, MATERIAL Y MÉTODOS}

Una vez obtenida la aprobación del proyecto por la Comisión de Investigación de nuestro centro y de la Junta Directiva de la Sociedad Española de Oftalmología se envió una encuesta confidencial de 30 preguntas a 276 centros oftalmológicos de España; 217 de ellos correspondientes a Hospitales del SNS, que se hizo llegar a principios del año 2005, a través de un envío postal.

De todas las preguntas, nueve hacían referencia a la atención de pacientes urgentes y además se plantearon dos situaciones clínicas hipotéticas (tabla I) de pacientes que acuden al hospital un viernes a las 3,30 de la tarde con un desprendimiento primario, producido por un desgarro situado en el meridiano de las XII, y sin afectación macular, efectuándose una serie de preguntas sobre cuándo debería ser intervenido de forma ideal, y cuándo lo sería probablemente en dicho hospital.

El análisis de los datos se realizó con el programa Statgraphics, versión Plus 5.1. (Manugistics, Inc, Dallas, EEUU). Para comprobar la representatividad de la muestra se utilizó un contraste de proporciones. Tras el análisis exploratorio de los datos se estudiaron las relaciones entre pares de variables de interés. La asociación entre las cuestiones cualitativas se evaluó a través del análisis de tablas de contingencia, utilizando el test chi-cuadrado con la corrección de Yates y el estadístico exacto de Fisher. Para las cuestiones cuantitativas, se utilizó el

\section{Tabla I. Preguntas sobre situaciones clínicas hipotéticas de pacientes que acuden al hospital con un desprendimiento primario}

Considere un paciente que se presenta en su servicio a las 3.30 $\mathrm{h}$ de un viernes, con un DR bulloso superior, un desgarro en herradura a las XII horas y la mácula pegada.

1. ¿En que período de tiempo el paciente debería, de forma ideal, ser intervenido?

2. Si en su servicio hay oftalmólogos dedicados a cirugía de vítreo retina, ¿cuándo sería operado el paciente?

3. Si en su servicio no hay oftalmólogos dedicados a cirugía de vítreo retina, ¿cuándo sería operado el paciente?

Considere un paciente con mácula pegada, DR superior bulloso, AV 1.0 en ese ojo, que se presenta a un servicio de oftalmología (no el suyo) a las 3,30 h de un viernes. El paciente es ingresado y es operado el lunes por la tarde, momento en el cual la AV ha disminuido a 0.1 a causa de la afectación de la mácula. La retina se reaplica, y no hay complicaciones, pero la AV no mejora más que 0.4 .

$¿$ Considera usted que el manejo del paciente ha sido el adecuado? contraste no paramétrico de Kruskall Wallis para la comparación de grupos y el coeficiente de correlación lineal de Pearson.

\section{RESULTADOS}

Se recibieron un total de 119 cuestionarios de hospitales del Sistema Nacional de Salud, lo que supone una tasa global de respuesta del 54,8\%. La mayor parte de ellas provinieron de hospitales docentes (HD) que constituyeron el 56,3\% del total, frente a los hospitales no docentes y comarcales, que fueron agrupados como $\mathrm{HnD}$.

Más del 80\% de los centros admitió tener problemas a la hora de atender de urgencia a un paciente con patología vitreo-retinina quirúrgica. Un análisis más detallado, reveló que aunque el $86 \%$ de los centros tiene acceso a quirófano de urgencia durante los fines de semana, sólo el 76,5\% pueden utilizar en ese periodo de tiempo el quirófano de oftalmología, con su dotación específica.

Mayor problema supone el contar con personal de enfermería entrenado para el manejo del equipamiento de vítreo-retina. El 82,5\% de los centros tiene acceso a enfermeras de urgencia en fines de semana, pero tan sólo el $21,9 \%$ lo tiene a enfermeras entrenadas en oftalmología. Sólo se encontraron diferencias estadísticamente significativas entre HD y $\mathrm{HnD}$ en cuanto a la utilización de quirófano de urgencias (Chi-cuadrado 7,21; p-valor 0,0072) y personal de urgencias (Chi-cuadrado 6,42; p-valor $0,0113)$ que fue mas frecuente en los HD.

Más de un $49 \%$ de los centros refiere encontrarse con dificultades medias o altas a la hora de acceder a los servicios de anestesia para tratar a este tipo de pacientes, y un $30 \%$ reconoce haber tenido que suspender y/o postergar alguna cirugía por falta de anestesista. No hubo diferencias entre HD y HnD.

Por último la tabla II, recoge la opinión de los oftalmólogos que rellenaron las encuestas acerca de cuales son los principales obstáculos para atender a los pacientes de urgencia. Sólo un $4 \%$ dijo que no tenía obstáculos, y no hubo diferencias entre los dos tipos de hospitales.

En el trabajo anterior se estableció que existe una media de 10,3 oftalmólogos por plantilla de hospital, de los que un $23 \%$ se dedica a la patología retiniana según declaran los que respondieron. La tabla 3 muestra que el 18\% de los centros carece de oftalmólogo de guardia, siendo este hecho más frecuen- 
Tabla II. Principales obstáculos para el manejo de desprendimientos de retina urgentes

\begin{tabular}{lrrr}
\hline & $\begin{array}{c}\text { Número } \\
\text { de centros }\end{array}$ & \multicolumn{1}{c}{$\%$} & $\%$ válidos \\
\hline Ninguno & 3 & $3,49 \%$ & $4,11 \%$ \\
Infraestructura & 40 & $46,51 \%$ & $54,79 \%$ \\
Personal & 24 & $27,91 \%$ & $32,88 \%$ \\
Infraestructura + Personal & 6 & $6,98 \%$ & $8,22 \%$ \\
Total & 73 & $84,88 \%$ & $100,00 \%$ \\
NS/NC & 13 & $15,12 \%$ & - \\
Total & 86 & $100,00 \%$ & - \\
\hline \hline
\end{tabular}

te en los $\mathrm{HnD}$ (Kruskal Wallis 16,06; p-valor $0,0001)$.

Además sólo en el 36,1\% de los centros los oftalmólogos de vítreo-retina cubren de forma permanente las urgencias de esta especialidad.

El 74\% de los centros que no disponen de especialistas de vítreo-retina de guardia, reconoce que tuvieron que derivar a los pacientes con DR de urgencia.

El 64\% de todos los centros afirmó que realizan vitrectomías (VPP), y de ellos el $77 \%$ que las realizan de urgencia. En ambos casos mayoritariamente en los HD.

En casi un $70 \%$ de los centros en los que se realizan VPP, se efectúan más de 100 cirugías programadas por año. Pero el número de las urgentes, cae a 10 VPP para los HD y 1,5 para los HnD por año (Kruskal Wallis 6,83; p-valor 0,0090).

Teniendo en cuenta el número de especialistas dedicados a la patología vitreoretiniana, puede establecerse que un retinólogo en un HD realiza unas 60 VPP programadas frente a las 24 de los $\mathrm{HnD}$. Y que la mediana de VPP urgentes por año es de 2,5 por especialista, con diferencias entre $\mathrm{HD}$ y $\mathrm{HnD}$ : 3,3 VPP urgentes al año en HD frente a 0,6 en los HnD (Kruskal Wallis 6,07; p-valor 0,0137).

Se planteó un caso hipotético en el que un paciente con un DR superior, bulloso, con un des- garro en herradura en el meridiano de las XII, y sin compromiso macular se presenta en el centro a las 3 de la tarde de un viernes, siendo la agudeza visual de 20/20 en ese momento. El 44\% de los centros (47 centros) respondieron que el paciente debería ser operado, de forma ideal, dentro de las primeras 24 horas. El 56\% restante (61 centros) señaló que el paciente debería ser operado a partir de las 24 horas del ingreso al centro. Sin embargo, tanto en los HD como en los HND, y en aquellos servicios con y sin especialistas en VR de guardia, reconocieron que más del $90 \%$ de pacientes con esas características deben esperar más de 24 horas para recibir tratamiento quirúrgico adecuado.

El $84 \%$ de los que respondieron a la encuesta consideraron que la demora en el tratamiento con la consiguiente caída de la visión a 20/80 por el compromiso de la mácula, se trata de una práctica inadecuada.

\section{DISCUSIÓN}

Una de las limitaciones de este trabajo ha sido la elección de la población de referencia. El catálogo de hospitales del SNS no recoge la información de los centros que cuentan con Servicio de Oftalmología y menos aún en los que existen especialistas en vítreo retina. Con la ayuda de una multinacional del sector y la colaboración de retinólogos de prestigio de cada Comunidad Autónoma se diseñó un listado que fue contrastado con el catálogo mencionado. La encuesta fue enviada a estos centros y dirigida al jefe de Servicio. Hay que resaltar que la encuesta estaba dirigida al jefe de servicio y no a los especialistas encargados de la patología vítreo-retiniana para los que está diseñado el cuestionario, por no contar tampoco con esa información.

Otro problema es la baja tasa de respuesta final, cercana al $48 \%$. Además, se observa que la distri-

Tabla III. Oftalmólogos de plantilla de guardia

\begin{tabular}{|c|c|c|c|c|c|c|c|c|c|}
\hline & \multicolumn{3}{|c|}{ Número de centros } & \multicolumn{3}{|c|}{$\%$} & \multicolumn{3}{|c|}{ \% válidos } \\
\hline & Total & HD & $\mathrm{HnD}$ & Total & HD & $\mathrm{HnD}$ & Total & HD & $\mathrm{HnD}$ \\
\hline 0 & 21 & 5 & 16 & $18,10 \%$ & $4,31 \%$ & $13,79 \%$ & $18,26 \%$ & $4,31 \%$ & $13,95 \%$ \\
\hline 1 & 82 & 51 & 31 & $70,69 \%$ & $43,97 \%$ & $26,72 \%$ & $71,30 \%$ & $43,97 \%$ & $27,33 \%$ \\
\hline 2 & 12 & 11 & 1 & $10,34 \%$ & $9,48 \%$ & $0,86 \%$ & $10,43 \%$ & $9,48 \%$ & $0,95 \%$ \\
\hline Total & 115 & 67 & 48 & $99,14 \%$ & $57,76 \%$ & $41,37 \%$ & $100,00 \%$ & $57,76 \%$ & $42,24 \%$ \\
\hline $\mathrm{NS} / \mathrm{NC}$ & 1 & 0 & 1 & $0,86 \%$ & $0,00 \%$ & $0,86 \%$ & - & - & - \\
\hline Total & 116 & 67 & 49 & $100,00 \%$ & $57,76 \%$ & $42,24 \%$ & - & - & - \\
\hline
\end{tabular}


bución de las respuestas no es uniforme. Puesto que el tipo de sondeo utilizado, por correo o por fax, característicamente presenta bajas tasas de respuesta, puede ser considerada como satisfactoria (9). Por otro lado, ésta resultó la única forma de llevar a cabo el proyecto.

Es importante mencionar varios sesgos que no deben ser subestimados. Uno de ellos es el «sesgo de la no respuesta» del que no se tiene información, al no haberse adoptado ningún mecanismo para conocerlo (10).

El otro, que en parte puede estar ligado al anterior, se refiere a la sobreestimación de las respuestas de los HD.

De cualquier forma, posteriores trabajos sobre el tema deberían tener en cuenta estos sesgos para reducir su influencia y hacer más representativos los resultados.

Existen otros problemas metodológicos que se refieren al cuestionario. No se sometió al mismo a la denominada fase de «pilotaje» ni se elaboraron instrucciones que incluyeran la definición de todos los conceptos que no se consideran estándar. Así, puede haber ocurrido que no todos los encuestados interpretaran de igual forma qué se entiende por «retinólogo» o por «estar de guardia».

Estas limitaciones han sido discutidas más extensamente en un artículo previo publicado sobre el Proyecto Retina 2, en donde se proponen a su vez algunas soluciones para evitarlas (8).

A pesar de éstas y otras posibles limitaciones, esta encuesta constituye el primer intento de obtención de datos, a nivel nacional, sobre el manejo de la patología vítreo-retiniana quirúrgica urgente y ha proporcionado algunos datos que parecen muy interesantes.

Teniendo en cuenta entonces esas limitaciones, es de destacar que más del $80 \%$ de los centros del SNS que respondieron a la encuesta refieren problemas a la hora de atender pacientes con patología vitreoretiniana quirúrgica de urgencia durante los fines de semana, atribuyendo la mayor parte de los centros las dificultades a problemas de infraestructura, y en menor medida a dificultades de personal. Dentro de esos problemas resulta interesante destacar que sólo un $22 \%$ de los centros cuenta con personal de enfermería entrenado en el manejo del complejo material de vítreo-retina. Además casi un $50 \%$ tiene problemas para que el Servicio de Anestesia facilite la cobertura imprescindible. Y sobre todo es importante destacar que sólo el 36\% de los centros tiene un retinólogo de guardia. Aún en esos centros, un paciente que acuda el viernes a primera hora de la tarde con un desprendimiento, no sería intervenido en las primeras 24 horas, según las respuestas recibidas.

Es sorprendente que si bien parece existir una distribución de los recursos a favor de los HD, ya sea médicos dedicados a VR o el acceso al personal entrenado en oftalmología durante los fines de semana, no se refleja esto por ejemplo, en una gran diferencia en cuanto al volumen de cirugías de urgencia realizadas en estos centros.

La Academia Americana de Oftalmología, no sólo enfatiza que si la mácula no está afectada debe repararse preferiblemente en las primeras 24 horas, sino que si hay amenaza de afectación macular, reduce el plazo a varias horas (4). Ho et al sólo encuentran una asociación significativa con la progresión a la afectación macular en la distancia del fluido subretiniano (FSR) a la fóvea (11). Sin embargo, el $61 \%$ de los pacientes que analizan fueron operados en el mismo día del inicio de los síntomas. Del 39\% restante, que recibió tratamiento más allá de las 24 horas, más de la tercera parte progresó a la afectación macular.

Por último el $84 \%$ de los encuestados españoles, reconoce que esta demora que puede afectar a la visión del paciente es una práctica inadecuada, lo que da una idea de que la demora de la cirugía que se observa en la mayoría de los centros que respondieron no responde a una mala práctica médica.

Puede deducirse por lo tanto que son los problemas de infraestructura y/o de organización los que impiden la cirugía de urgencia. Queda en evidencia que existe una falta de coordinación de los recursos, o una administración ineficiente de los mismos, ya que si bien en el 36\% de los centros hay especialista de VR de guardia, el 78\% de los centros en donde se realizan VPP no podrá acceder a personal capaz de instrumentar la cirugía.

Cierta similitud en esta situación se encontraba en el Reino Unido hace 6 años (7). Aunque muchos hospitales, sobre todo los docentes, tenían acceso tanto a quirófanos especializados de Oftalmología como a personal de enfermería específicamente entrenado, en la realidad se producían demoras atribuidas por una parte a que sólo un $28 \%$ de los cirujanos de vítreo-retina estaban de guardia y a que un buen número de los «fellows» de vítreo-retina, que están en los hospitales docentes, no estaban formalmente localizados. Los resultados de esta encuesta 
sirvieron para introducir cambios en el Sistema Nacional de Salud británico con vistas a garantizar un tratamiento quirúrgico más adecuado a los pacientes con desprendimientos de retina.

Otro dato interesante es el escaso número de vitrectomías de urgencia que se practican, de tan sólo 10 anuales en los HD y de 1,5 en los HnD. Parece innecesario discutir que con tan bajo número de urgencias no se justifica el dotar a cada hospital de recursos necesarios para realizar esta cirugía de urgencia y que estos datos son argumentos a favor de concentrar la atención de estas urgencias en determinados centros, tal y como se ha realizado en el Reino Unido.

A pesar de que los datos obtenidos en este trabajo deben analizarse con precaución por la manera en la que se seleccionó la muestra y por no haber tenido en cuenta el sesgo de la no-respuesta, se han podido identificar algunas de las principales deficiencias que sufre hoy el SNS español en el manejo de los desprendimientos de retina de urgencia y los recursos con los que cuenta. Estos datos pueden ser de gran interés para las autoridades sanitarias si se desea organizar una cobertura apropiada y un modelo más eficiente de atención de las urgencias de retina en un futuro.

\section{AGRADECIMIENTO}

A todos los jefes de servicio de los hospitales y clínicas oftalmológicas españolas. A la Junta Directiva de la Sociedad Española de Oftalmología y a Novartis SA.

\section{BIBLIOGRAFÍA}

1. Duke-Elder S, Dobree JH. Detachment and folding of the retina. In: Duke-Elder S, Dobree JH. System of Ophthalmology. Diseases of the retina. St Louis: Mosby-Year Book; 1967; 771.

2. Sullivan PM, Luff AJ, Aylward GW. Results of primary retinal reattachment surgery: a prospective audit. Eye 1997; 11: 869-871.

3. Hassan TS, Sarrafizadeh R, Ruby AJ, Garretson BR, Kuczynski B, Williams GA. The effect of duration of macular detachment on results after the scleral buckle repair of primary, macula-off retinal detachments. Ophthalmology 2002; 109: 146-152.

4. The repair of rhegmatogenous retinal detachments. American Academy of Ophthalmology. Ophthalmology 1996; 103: 1313-1324.

5. Michels RG, Wilkinson CP, Rice TA. Results of retinal reattachment surgery. In: Michels RG, Wilkinson CP, Rice TA. Retinal detachment. St. Louis: Mosby; 1990; 917-958.

6. Patel NN, Bunce C, Asaria RH, Charteris DG. Resources involved in managing retinal detachment complicated by proliferative vitreoretinopathy. Retina 2004; 24: 883-887.

7. Frimpong-Ansah S, Kirkby GR. Arrangements for the management of urgent retinal detachments in the United Kingdom and Eire in year 2000: results of a survey. Eye 2002; 16: 754-760.

8. Fernández I, Rojas J, Pastor JC, Gómez-Ulla F, Piñero A. Encuesta nacional sobre la cirugía vitreo-retiniana y el manejo de los desprendimientos de retina regmatógenos en España. Proyecto Retina 2. Arch Soc Esp Oftalmol 2006; 81:635-640.

9. McColl E, Jacoby A, Thomas L, Soutter J, Bamford C, Steen $N$, et al. Design and use of questionnaires: a review of best practice applicable to surveys of health service staff and patients. Health Technol Assess 2001; 5: 1-256.

10. Rao RS, Glickman ME, Glynn RJ. Use of covariates and survey wave to adjust for nonresponse. Biom J 2004; 46: 579-588.

11. Ho SF, Fitt A, Frimpong-Ansah K, Benson MT. The management of primary rhegmatogenous retinal detachment not involving the fovea. Eye 2006; 20: 1049-1053. 